\title{
Preservation of the bladder, but at what cost?
}

\author{
Niklas Harland, Arnulf Stenzl \\ Department of Urology, Eberhard-Karls-University, Tübingen, Germany \\ Correspondence to: Arnulf Stenzl. Department of Urology, University Hospital Tübingen, Hoppe-Seyler-Straße 3, Tübingen 72076, Germany. \\ Email: arnulf.stenzl@med.uni-tuebingen.de. \\ Provenance: This is an invited article commissioned by the Section Editor Xiao Li (Department of Urology, Jiangsu Cancer Hospital \& Jiangsu \\ Institute of Cancer Research \& Nanjing Medical University Affiliated Cancer Hospital, Nanjing, China). \\ Comment on: Williams SB, Shan Y, Ray-Zack MD, et al. Comparison of Costs of Radical Cystectomy vs Trimodal Therapy for Patients With \\ Localized Muscle-Invasive Bladder Cancer. JAMA Surg 2019. [Epub ahead of print].
}

Submitted Aug 22, 2019. Accepted for publication Sep 22, 2019.

doi: $10.21037 /$ tau.2019.09.40

View this article at: http://dx.doi.org/10.21037/tau.2019.09.40

Radical cystectomy ( $\mathrm{rCx}$ ) with pelvic lymphadenectomy is the gold standard for muscle invasive bladder cancer. Nevertheless advantages of trimodal treatment (TMT) over $\mathrm{rCx}$ is an ongoing discussion in urology. According to the guidelines of the European Association of Urology and the SIU, TMT should only be offered to "selected, well-informed, compliant" patients $(1,2)$. Due to a lack of randomized studies comparing the two, we can only rely on retrospective analysis. Most of these focus on the functional and oncological outcome. With aging populations in most industrialized countries and therefore rising healthcare expenses, cost effectiveness is of growing importance. This is reflected in a trend in current publications to investigate the differences in cost of different treatments available. However, as stated in the article this does not mean costeffectiveness, i.e., oncological outcome and quality of life.

In a recently published manuscript, Williams et al. compared the cost of rCx and TMT in the USA (3). For this purpose, they analysed data extracted from the Medicare database of 2,235 and 728 patients undergoing $\mathrm{rCx}$ and TMT, respectively. All expenditures within one year after diagnosis of muscle invasive disease were combined and compared at different time points. $\mathrm{rCx}$ was associated with lower cost at 90, 180 and 365 days, with growing difference over time. At one year, the cost for patients undergoing TMT was almost twice as much as for patients undergoing $\operatorname{rCx}(\$ 289,142$ vs. \$148,757).

This study is an important addition to the discussion about the best approach to muscle invasive bladder cancer. While the best oncological and functional outcome should always be the primary goal for all decisions in medicine, we should not lose sight of the cost. We applaud the authors for looking at this important question. The study has some limitations that one needs to be aware of when looking at the results.

Firstly, the fact, that the difference in cost was rising within the first year suggests, that this trend might continue in the future. After bladder sparing treatment there is a relevant risk of local recurrence of $33 \%$ within two years and $11 \%$ relapse with muscle invasive disease by this time (4). The possibility of late recurrence causes the need for extensive follow-up and in many cases repeated transurethral resection or even salvage $\mathrm{rCx}$. The high numbers in the timeframe of two years suggests, that we need to look at longer intervals to find the real difference in cost.

The need for prospective data is further underscored by a systematic failure that comes with the approach chosen by Williams et al. To define patients receiving TMT they included patients undergoing transurethral resection of bladder tumor, radiation and chemotherapy but excluded those, that additionally underwent $\mathrm{rCx}$. This is problematic, because we know that about $11 \%$ of patients will relapse with muscle invasive bladder cancer within 2 years after TMT.

The recommended treatment in this situation is salvage $\mathrm{rCx}$. Therefore, including patients who received chemotherapy, radiation and $\mathrm{rCx}$ into the cystectomy group will wrongly assign the most costly group of TMT to rCx.

Furthermore, we don't know much about the clinical data, it is therefore impossible to know how many of the 
patients undergoing $\mathrm{rCx}$ would have been eligible for TMT. It is possible that the $\mathrm{rCx}$ group included the patients with complicating conditions, i.e., higher tumor stage, hydronephrosis and chronic kidney failure. This would again lead to an underestimation of the cost difference.

In summary, this article adds urgently needed knowledge to a highly discussed topic. While many groups have discussed oncological and functional outcomes, little is known about the cost associated with each treatment approach. As elaborated above, it is reasonable to assume, that the difference between both groups is even higher than calculated in this study. For future decision making we eagerly await the results of several currently ongoing trials investigating the effect of immune-checkpoint inhibitors on TMT (i.e., NCT02662062, NCT02621151). While this might improve the tolerability and the necessary oncological outcome of the treatment, it will surely further increase the cost associated with it.

\section{Acknowledgments}

None.

\section{Footnote}

Conflicts of Interest: A Stenzl is a member of the advisory board for Ipsen Pharma, Roche, Janssen, Alere, Bristaol
Myers Squibb, Stebabiotech, Synergo and Ferring. $\mathrm{He}$ was a speaker for Janssen, Ipsen Pharma, Sanofi Aventis, CureVac, Astellas and Amgen. He received research grants by Amgen, immatics biotechnologies, Novartis and Karl Storz. N Harland has no conflicts of interest to declare.

Ethical Statement: The authors are accountable for all aspects of the work in ensuring that questions related to the accuracy or integrity of any part of the work are appropriately investigated and resolved.

\section{References}

1. Alfred Witjes J, Lebret T, Compérat EM, et al. Updated 2016 EAU guidelines on muscle-invasive and metastatic bladder cancer. Eur Urol 2017;71:462-75.

2. Leow JJ, Bedke J, Chamie K, et al. SIU-ICUD consultation on bladder cancer: treatment of muscleinvasive bladder cancer. World J Urol 2019;37:61-83.

3. Williams SB, Shan Y, Ray-Zack MD, et al. Comparison of Costs of Radical Cystectomy vs Trimodal Therapy for Patients With Localized Muscle-Invasive Bladder Cancer. JAMA Surg 2019:e191629. [Epub ahead of print].

4. James ND, Hussain SA, Hall E, et al. Radiotherapy with or without chemotherapy in muscle-invasive bladder cancer. N Engl J Med 2012;366:1477-88.
Cite this article as: Harland N, Stenzl A. Preservation of the bladder, but at what cost? Transl Androl Urol 2019;8(Suppl 5):S474-S475. doi: 10.21037/tau.2019.09.40 